\title{
Microbiome Analysis from Paired Mucosal and Fecal Samples of a Colorectal Cancer Biobank
}

\author{
Ulrich Wirth ${ }^{1}$, Debora Garzetti ${ }^{2,3}$, Lara M. Jochum ${ }^{2}$, Stefanie Spriewald ${ }^{2}$, Florian Kühn ${ }^{1}$, \\ Matthias Ilmer 1,4 (D), Serene M. L. Lee ${ }^{1,5}$, Hanno Niess 1,5 , Alexandr V. Bazhin ${ }^{1,4}$, \\ Joachim Andrassy 1,4, Jens Werner 1,4, Barbara Stecher ${ }^{2,3}$ and Tobias S. Schiergens 1,4,5,* (D) \\ 1 Department of General, Visceral and Transplant Surgery, Ludwig-Maximilians-University Munich, \\ Marchioninistr. 15, D-81377 Munich, Germany; Ulrich.Wirth@med.uni-muenchen.de (U.W.); \\ Florian.Kuehn@med.uni-muenchen.de (F.K.); Matthias.Ilmer@med.uni-muenchen.de (M.I.); \\ Serene.Lee@med.uni-muenchen.de (S.M.L.L.); Hanno.Niess@med.uni-muenchen.de (H.N.); \\ Alexandr.Bazhin@med.uni-muenchen.de (A.V.B.); Joachim.Andrassy@med.uni-muenchen.de (J.A.); \\ Jens.Werner@med.uni-muenchen.de (J.W.) \\ 2 Max-Von-Pettenkofer Institute, Ludwig-Maximilians-University Munich, Pettenkoferstr. 9A, \\ D-80336 Munich, Germany; Debora.garzetti@gmail.com (D.G.); Laramjochum@gmail.com (L.M.J.); \\ Sj.spriewald@gmx.de (S.S.); stecher@mvp.lmu.de (B.S.) \\ 3 Center for Infection Research (DZIF), Partner Site Munich, Pettenkoferstr. 9A, D-80336 Munich, Germany \\ 4 German Cancer Consortium (DKTK), Partner Site Munich, Marchioninistr. 15, D-81377 Munich, Germany \\ 5 Biobank under the Administration of the Human Tissue and Cell Research (HTCR), Marchioninistr. 15, \\ D-81377 Munich, Germany \\ * Correspondence: tobias.schiergens@med.uni-muenchen.de; Tel.: +49-89-4400-711226; \\ Fax: +49-89-4400-76574
}

Received: 11 November 2020; Accepted: 8 December 2020; Published: 9 December 2020

Simple Summary: The role of gut microbiota in colorectal cancer is subject to extensive research. The aim of this study was to assess the feasibility of DNA extraction and microbiome profiling of samples from different sample sites, tissue sites and storage duration of a colorectal cancer biobank. Mucosa samples, mucosal scrapings and feces as well as different tissue sites (tumor, normal mucosa) were analyzed. Microbiome analysis could be successfully performed in most of the samples (overall 93.3\%) with sufficient numbers of high-quality reads. There were no differences between sample sites, while in some measures, significant differences were found between tumor and normal mucosa. Samples stored for up to eight years were used and storage conditions had no significant influence on the results. Microbiome analysis can be carried out successfully in fecal, normal mucosal and tumor samples stored long term in a colorectal cancer biobank, hence large retrospective microbiome association studies are feasible.

Abstract: The role of gut microbiota in colorectal cancer is subject to extensive research. Before usage
of biorepositories for microbiome studies, it is crucial to evaluate technical feasibility of microbiome
profiling from various biospecimens. The aim of this study was to assess the feasibility of DNA-
extraction and microbiome profiling of samples from different sample sites, tissue sites and storage
duration of a colorectal cancer biobank. Mucosa samples, mucosal scrapings and feces as well as
different tissue sites (tumor, normal mucosa) were analyzed. $16 \mathrm{~S}$ rRNA gene-based microbiome
profiling with taxonomic assignment was performed on the Illumina MiSeq (Illumina, San Diego,
USA) platform from stored snap frozen samples. For statistical analysis, $\alpha$ - and $\beta$-diversity measures,
PCoA, permutational multivariate analysis of variance and graphical representation were performed.
Microbiome analysis could be successfully performed in most of the samples (overall $93.3 \%$ with
sufficient numbers of high-quality reads. There were no differences between sample sites, while in
some measures significant differences were found between tumor and normal mucosa ( $\alpha$-diversity,
Shannon/Simpson Indices $p=0.028 / 0.027$, respectively). Samples stored for up to eight years were 
used and storage conditions had no significant influence on the results. Tumor and tissue samples of a biobank stored long term can be successfully used for microbiome analysis. As large sample sizes are needed for association studies to evaluate microbial impact on tumorigenesis or progression of colorectal cancer, an already established biorepository may be a useful alternative to prospective clinical studies.

Keywords: gut microbiome; 16S rRNA; colorectal cancer; biobank; biorepository

\section{Introduction}

In recent years, the relevance of sequence-based analysis of the gut microbiota in patients with various diseases has risen sharply [1]. Association studies have linked alterations of the gut microbiota with a variety of human diseases [2-8]. The abundance of different microbiota also varies highly among healthy individuals depending on environmental conditions, genetics, the host's immune system, diet as well as infections or use of antibiotics $[9,10]$. In colorectal cancer (CRC), there is an increasing evidence suggesting that the gut microbiota are associated with CRC development [11,12]. Gut microbiota show significant impact on processes involved in cellular DNA damage, DNA methylation, chromatin structure modulation and non-coding RNA expression [1]. Furthermore, they play distinct roles in pathways of cell proliferation such as WNT signaling [1,11]. Fusobacterium nucleatum was causally linked with tumorigenesis of sporadic CRC [13]. Furthermore, Escherichia coli strains harboring a genomic virulence island ( $p k s)$ can cause DNA damage and chromosomal instability in the host [14]. In addition, next-generation sequencing technologies also revealed the relevance of other CRC-associated gut bacteria such as Bacteroides fragilis, Escherichia coli, Parvimonas micra, Peptostreptococcus stomatis and Atobobium parvulum [11]. According to the oral-gut hypothesis of CRC tumorigenesis, several of these bacteria, Fusobacterium nucleatum, Parvimonas micra and Peptostreptococcus stomatis in particular, originate from the oral cavity and are opportunistic colonizers of the colon reflecting intestinal dysbiosis $[15,16]$. Despite evident regional differences in microbial communities [17], Wirbel et al. could identify 29 microbial species consistently associated with CRC tumorigenesis throughout different countries [12]. Microbial signatures were even shown to improve sensitivity of CRC prediction when combined with fecal occult blood testing (FOBT) by $>45 \%$ relative to FOBT alone [18]. In addition, microbial pathogens, such as Enterobacteriaceae, Enterococcus spp., Staphylococcus spp. and Pseudomonas aeruginosa have been shown to be associated with surgical complications, especially surgical site infections and anastomotic leakage in patients undergoing colorectal resections $[10,19]$. Hence, a better understanding of the gut microbiome might play an important role to improve diagnostics and therapy of CRC patients.

For researchers, the opportunity to analyze not only biospecimens within prospective or cross-sectional studies but also the chance to use large biorepositories would enable meaningful translational studies further investigating the role of the gut microbiota in CRC. For this purpose, biobanks can provide important resources, for example tumor and adjacent normal tissue samples, blood, patient-derived in vitro cell models as well as the corresponding clinical data. Identifying distinct phenotypes including the microbial profile could help to develop tailored diagnostic as well as therapeutic strategies depending on the individual risk for perioperative complications and cancer progression.

To enable the use of large colorectal cancer biorepositories for microbiome association studies, this work aimed to investigate the feasibility of microbiome analysis in snap frozen samples from a colorectal cancer biobank collected from different sample sites (mucosal, luminal), tissue sites (tumor, normal mucosa) and with varying storage durations. 


\section{Results}

\subsection{Microbiome Profiling of Luminal and Mucosal Sample Sites (Step 1)}

The procedural characteristics from samples of step 1 experiments are shown in Table 1.

Table 1. Procedural characteristics of samples collected from different sample (Step 1 experiments) and tissue sites as well as storage duration (Step 2 experiments).

\begin{tabular}{|c|c|c|c|c|c|}
\hline & $\begin{array}{c}\text { Sample } \\
\text { Collection } \\
\text { Site }\end{array}$ & $\begin{array}{l}\text { Weight (g) of } \\
\text { Samples } \\
\text { (Mean) }\end{array}$ & $\begin{array}{l}\text { IT }^{1}(\min ) \mathrm{OR}^{2} \\
\text { until Sampling } \\
(\text { Mean } \pm \text { SD) }\end{array}$ & $\begin{array}{c}\text { IT }^{1}(\min ) \text { Sampling } \\
\text { until Freezing } \\
(\text { Mean } \pm \text { SD) }\end{array}$ & $\begin{array}{l}\text { Duration (Months) } \\
\text { of Storage at }-80^{\circ} \mathrm{C} \\
\text { (Mean } \pm \text { SD) }\end{array}$ \\
\hline $\begin{array}{c}\text { Step } 1 \\
n=6\end{array}$ & $\begin{array}{c}\text { Feces } \\
\text { Mucosa } \\
\text { Scraping }\end{array}$ & $\begin{array}{l}0.19 \\
0.23 \\
0.36\end{array}$ & $36.3 \pm 23.8$ & $114.3 \pm 95.6$ & $4.0 \pm 1.3$ \\
\hline $\begin{array}{c}\text { Step } 2 \\
n=6\end{array}$ & $\begin{array}{l}\text { Mucosa } \\
\text { Tumor }\end{array}$ & $\begin{array}{l}0.20 \\
0.26\end{array}$ & $39.2 \pm 20.3$ & $37.8 \pm 10.0$ & $59.0 \pm 37.6$ \\
\hline
\end{tabular}

The samples were stored for an average time of $4.0 \pm 1.3$ months. Microbiome analysis could be successfully performed in five out of six fecal samples, five out of six mucosa samples and six out of six mucosal scraping samples (16 out of 18 samples overall, $89 \%$ ) with a sufficiently high amplicon DNA concentration. Only in one fecal and one tissue sample, the amplicon DNA concentration was quite low despite a high DNA concentration after extraction (presumably human DNA). DNA yield after extraction and amplification as well as high-quality reads of samples are given in Table 2.

Table 2. DNA, amplicon yield, amount of high-quality reads and $\alpha$-diversity measures in samples collected from different compartments (Step 1).

\begin{tabular}{|c|c|c|c|c|c|c|c|c|}
\hline & & \multicolumn{3}{|c|}{ Basic Data } & \multicolumn{4}{|c|}{$\alpha$-Diversity Measures } \\
\hline & & $\begin{array}{c}\text { gDNA }(\mathrm{ng} / \mu \mathrm{L}) \\
\text { (PicoGreen) }\end{array}$ & $\begin{array}{c}\text { DNA } \\
\text { Concentration } \\
\text { (ng/ } / \mu \mathrm{L}) \text { after } \\
\text { Amplification }\end{array}$ & Reads/Sample & Chao1 & ACE & Shannon & Simpson \\
\hline \multirow{8}{*}{ Feces } & 1 & 103.88 & 9.95 & 38,830 & \multirow{8}{*}{3848} & \multirow{7}{*}{4094} & \multirow{7}{*}{3.59} & \multirow{7}{*}{0.93} \\
\hline & 2 & 186.72 & 7.98 & 61,629 & & & & \\
\hline & 3 & 143.18 & 4.23 & 4733 & & & & \\
\hline & 4 & 163.34 & 3.26 & 54,584 & & & & \\
\hline & 5 & 165.46 & 0.04 & 249 * & & & & \\
\hline & 6 & 116.20 & 6.32 & 74,427 & & & & \\
\hline & Mean \pm SD & $146.5 \pm 31.6$ & $5.30 \pm 3.50$ & $39,075 \pm 30,613$ & & & & \\
\hline & 1 & 182.15 & 3.17 & 46,077 & & \multirow{6}{*}{1163} & \multirow{6}{*}{3.62} & \multirow{6}{*}{0.93} \\
\hline \multirow{5}{*}{ Mucosa } & 2 & 172.71 & 0.27 & $173 *$ & \multirow{5}{*}{1207} & & & \\
\hline & 3 & 174.29 & 3.60 & 6058 & & & & \\
\hline & 4 & 167.36 & 5.82 & 8294 & & & & \\
\hline & 5 & 179.41 & 7.84 & 13,265 & & & & \\
\hline & 6 & 171.14 & 5.32 & 38,225 & & & & \\
\hline \multirow{8}{*}{ Scraping } & Mean \pm SD & $174.51 \pm 5.45$ & $4.34 \pm 2.60$ & $59,397 \pm 14,974$ & \multirow{8}{*}{6165} & \multirow{8}{*}{6064} & \multirow{8}{*}{4.08} & \multirow{8}{*}{0.95} \\
\hline & 1 & 18.95 & 7.25 & 62,021 & & & & \\
\hline & 2 & 42.91 & 1.68 & 45,152 & & & & \\
\hline & 3 & 8.14 & 6.65 & 50,863 & & & & \\
\hline & 4 & 118.70 & 8.98 & 84,086 & & & & \\
\hline & 5 & 6.86 & 10.18 & 46,675 & & & & \\
\hline & 6 & 6.04 & 4.33 & 67,589 & & & & \\
\hline & Mean \pm SD & $33.60 \pm 43.97$ & $6.51 \pm 3.11$ & $18,682 \pm 18,823$ & & & & \\
\hline
\end{tabular}

* Failed samples.

The mean DNA concentration of all samples after 16S rRNA gene amplification before sequencing was $5.38 \pm 3.06 \mathrm{ng} / \mu \mathrm{L}$ (range: $0.04-10.18 \mathrm{ng} / \mu \mathrm{L}$ ). After sequencing and quality control, the mean number of high-quality reads was 39,052 $\pm 27,173$ counts/sample (range: 173-84,086 counts/sample). Because of a low number of high-quality reads in the above described fecal and tissue sample (173 and 
249 reads/sample, respectively), these two samples were excluded from further analysis. In the remaining samples, 702,508 16S rRNA gene sequences could be obtained in total and sub-sampling (rarefaction) was performed to the size of the smallest amount of counts/sample $(n=4733)$ for each sample. There was a moderate inverse correlation between the number of obtained high quality reads per sample and concentration of genomic DNA after extraction $(\mathrm{r}=-0.476 ; p=0.046)$ as well as a moderate correlation to the DNA concentration after amplification $(r=0.477 ; p=0.045)$.

The $\alpha$-diversity measures for mucosa samples, mucosal scrapings and feces of step 1 experiments are presented in Table 2 as well as Figure 1A,B. Wilcoxon rank sum test showed no significant difference between groups for $\alpha$-diversity measures (Chao1 and ACE Richness, Shannon and Simpson Indexes; $p>0.05$, pairwise comparison).
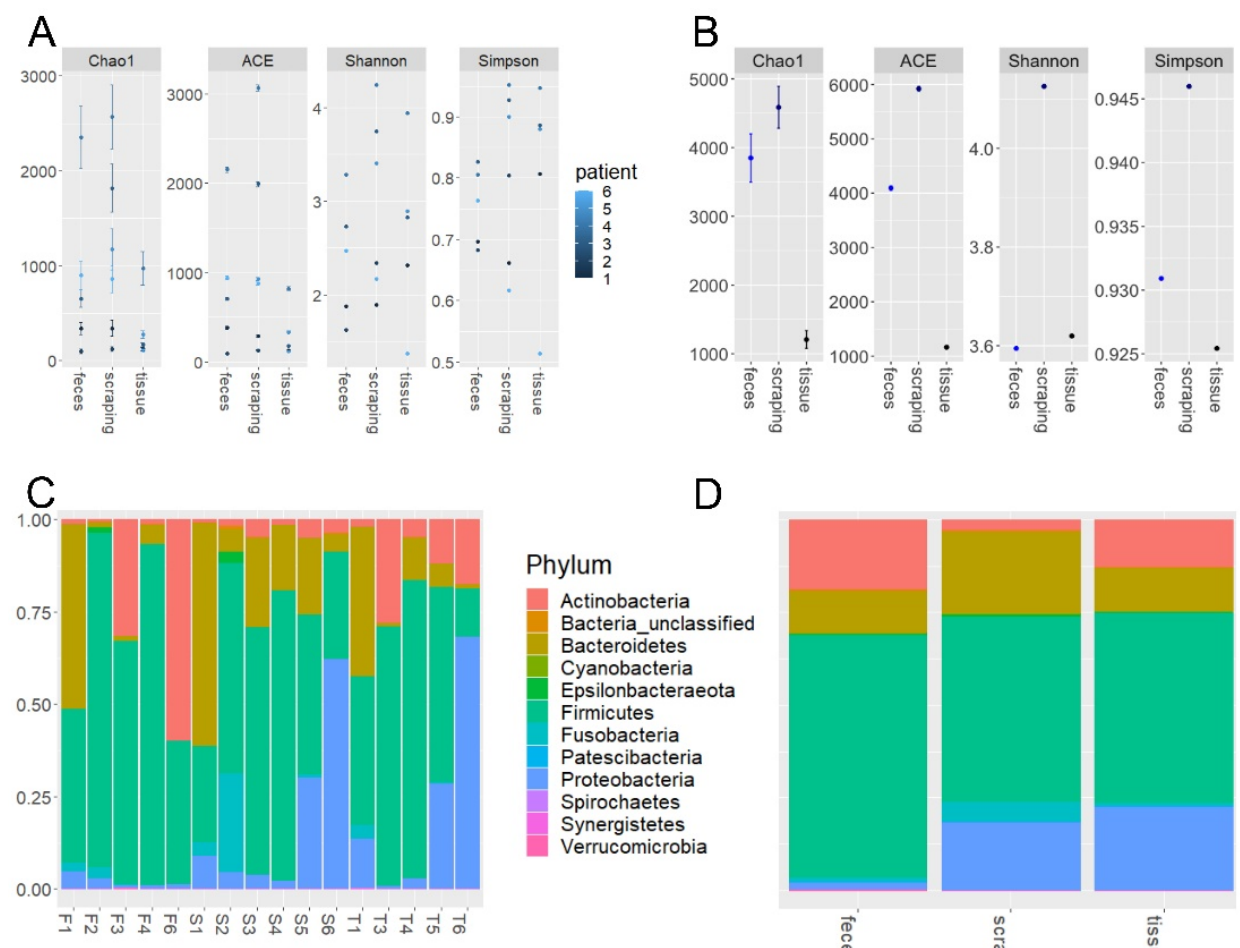

$\mathrm{D}$
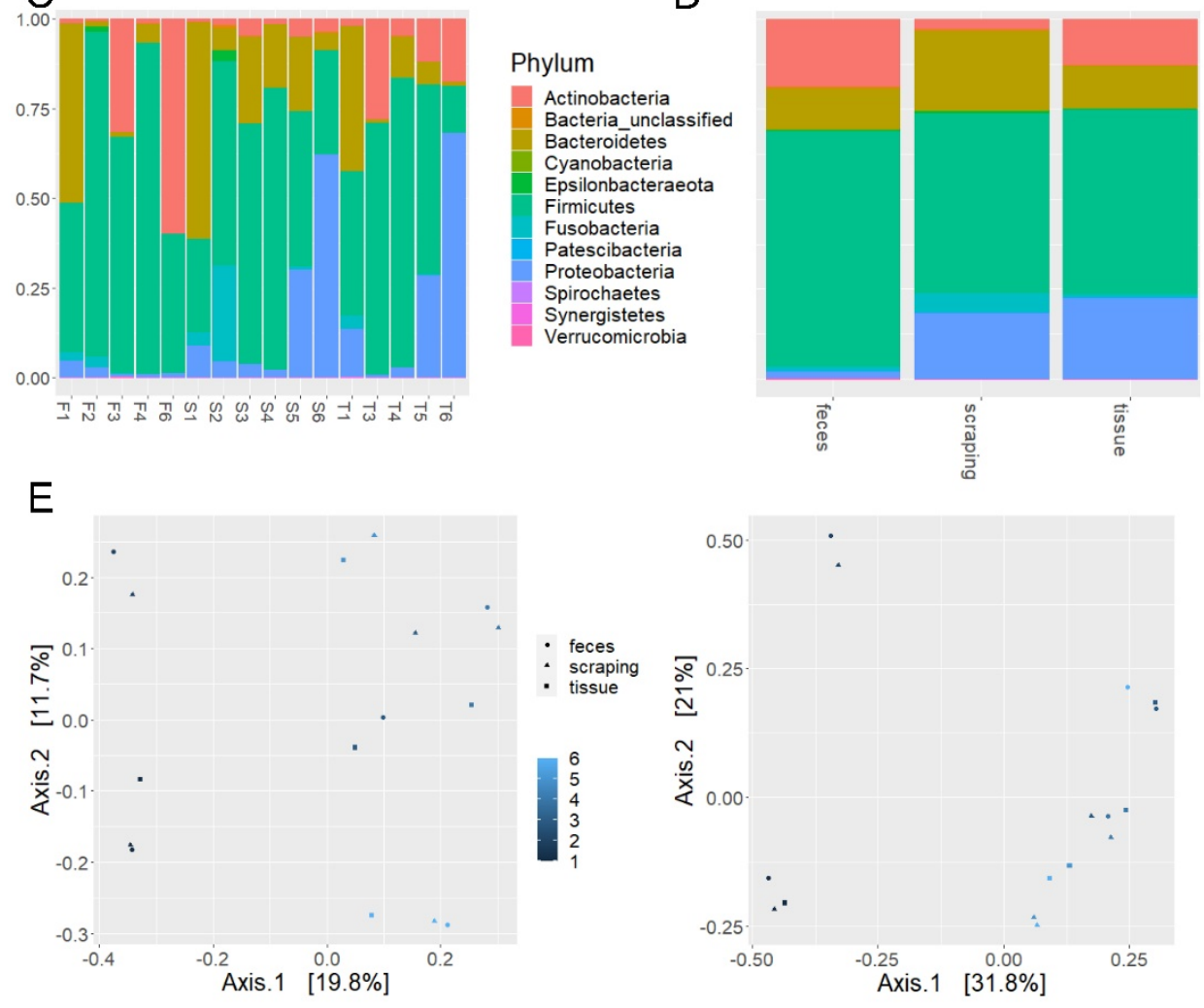

Figure 1. Alpha-diversity measures for all samples from (A) feces, scrapings as well as mucosa tissue and (B) between sample collection sites of trial step 1 experiments. Abundance on phylum level for (C) all individual samples from feces [F], scrapings [S], mucosa tissue [T] and (D) the same samples stratified according to sample site (number of reads normalized using median sequencing depth). Principal coordinate analysis based on weighted (E) and un-weighted (F) UniFrac distances for fecal samples, tissue samples and mucosal scrapings. 
Predominant phyla were Firmicutes, Bacteroidetes, Proteobacteria, Actinobacteria and Fusobacteria (Table 3).

Table 3. Relative abundance for most abundant species on phylum level (\%) in step 1 experiments.

\begin{tabular}{cccc}
\hline Species & Feces & Scrapings & Mucosa \\
\hline Firmicutes & 65.8 & 50.4 & 51.4 \\
Bacteroidetes & 11.6 & 22.1 & 12.1 \\
Proteobacteria & 2.1 & 18.5 & 22.7 \\
Actinobacteria & 18.9 & 2.9 & 12.9 \\
Fusobacteria & 1.1 & 5.2 & 0.8 \\
\hline
\end{tabular}

The relative abundance for the different samples based on phylum levels is shown in Figure 1C,D. The relative distribution of phyla showed variations to some extent, but due to small sample size no comparative analysis were performed. Weighted and unweighted UniFrac analyses showed no visual distinction between groups representing luminal and mucosal compartments (feces to scraping 0.43/0.74; feces to mucosa 0.36/0.69; scraping to mucosa 0.31/0.73) as shown by PCoA in Figure 1E,F. This could be confirmed by PERMANOVA (weighted UniFrac: $\mathrm{R} 2=0.106 ; p=0.925$; unweighted UniFrac: $\mathrm{R} 2=0.107 ; p=0.681)$.

\subsection{Microbiome Profiling from Tumor and Adjacent Normal Mucosa Samples as Well as Influence of Storage Duration (Step 2)}

The procedural characteristics from samples of Step 2 experiments are shown in Table 1. The samples were stored for an average time of $58.5 \pm 37.6$ months. Microbiome analysis could be successfully performed with a sufficient amount of high-quality reads $(836,791$ 16S rRNA gene sequences) in all six tumor and six corresponding mucosa tissue samples (12 out of 12, 100\%) (Table 4).

Table 4. Amount of high-quality reads and $\alpha$-diversity measures as well as relative abundance for most abundant species on phylum level (\%) of tumor and normal mucosa tissue samples in Step 2 experiments.

\begin{tabular}{cccc}
\hline & & Mucosa & Tumor \\
\hline \multirow{2}{*}{ Reads/sample \pm SD } & $70,621 \pm 30,690$ & $68,843 \pm 29,020$ \\
\hline \multirow{3}{*}{-diversity measures } & Chao1 & 12,465 & 8553 \\
& ACE & 13,690 & 9191 \\
& Shannon & 4.85 & 3.80 \\
& Simpson & 0.98 & 0.91 \\
\hline \multirow{2}{*}{ Relative abundance } & Firmicutes & 80.5 & 46.3 \\
& Proteobacteria & 10.9 & 38.6 \\
& Bacteroidetes & 3.6 & 5.9 \\
& Actinobacteria & 2.6 & 1.8 \\
& Fusobacteria & 0.6 & 5.2 \\
\hline
\end{tabular}

Sub-sampling (rarefication) was performed to the smallest number of counts/sample $(n=15,147)$ for each sample. There was no relevant correlation between number of high-quality reads and duration of storage at $-80^{\circ} \mathrm{C}(\mathrm{r}=0.221 ; p=0.490)$ or weight of the used samples $(\mathrm{r}=0.123 ; p=0.704)$.

Comparing the $\alpha$-diversity measures between mucosal and tumor site samples, no significant differences between groups for Chao1 $(p=0.249)$ or ACE $(p=0.249)$ richness-based measures was observed; for Shannon and Simpson indices, respectively, there were significant differences between groups ( $p=0.028$ and $p=0.027$, respectively, Wilcoxon rank sum test; Table 4 , Figure 2A,B). 

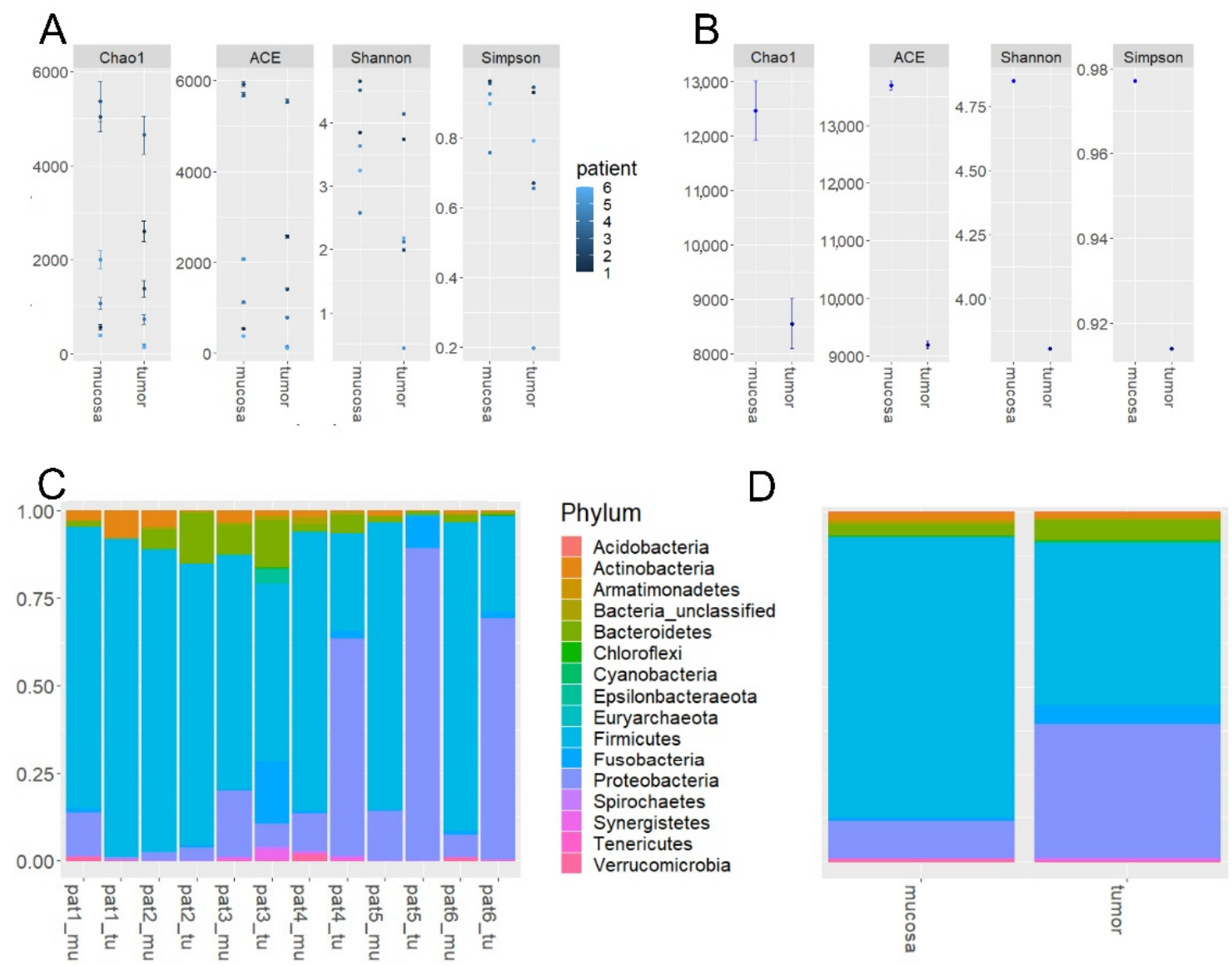

E

F
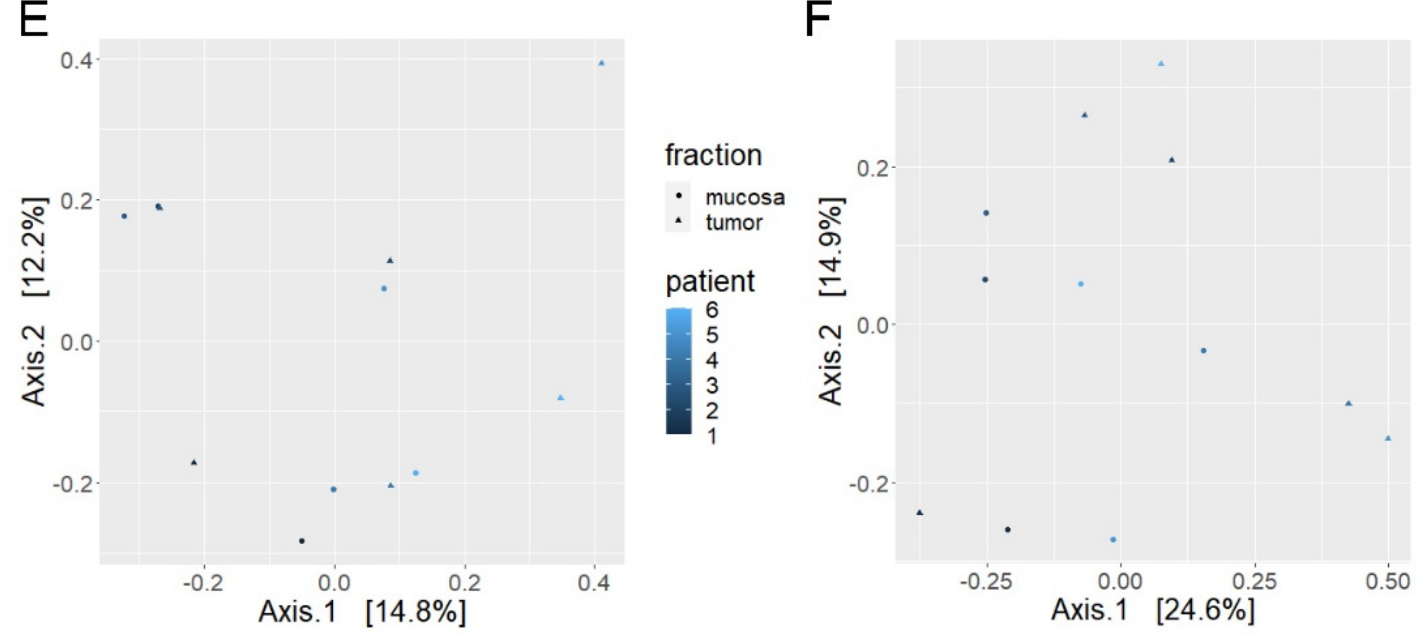

Figure 2. Alpha-diversity measures for all samples from (A) normal mucosa and tumor tissue as well as (B) between tumor and normal mucosa (mean) of step 2 experiments. Abundance on phylum level for (C) all individual patient samples [pat] and (D) the same samples grouped as tumor or mucosa tissue (number of reads normalized using median sequencing depth; tu = tumor sample; $\mathrm{mu}=$ mucosa sample). Principal coordinate analysis based on weighted (E) and un-weighted (F) UniFrac distances for mucosa and tumor samples.

Predominant phyla are shown in Table 4 and Figure 2C,D. Actinobacteria were significantly more abundant in normal mucosa samples compared to tumor tissue ( $p=0.028$, Wilcoxon rank sum test). Beyond that, a variation in the relative distribution of phyla between mucosa and tumor samples was observed to some extent without significance with e.g., a higher proportion of Fusobacteria in tumor tissue samples as compared to mucosa samples. 
Weighted and unweighted UniFrac analyses showed no visual distinction between tumor and normal mucosa samples representing the mucosal compartment (mucosa to tumor 0.795/0.528) as shown using PCoA in Figure 2E,F. This was confirmed by PERMANOVA (weighted UniFrac: R2 = 0.0831 ; $p=0.758$; unweighted UniFrac: $\mathrm{R} 2=0.10783 ; p=0.263$ ).

The abundance of mucosa and tumor samples is also illustrated as heatmap (Figure 3) and krona diagrams (Figure 4A,B).

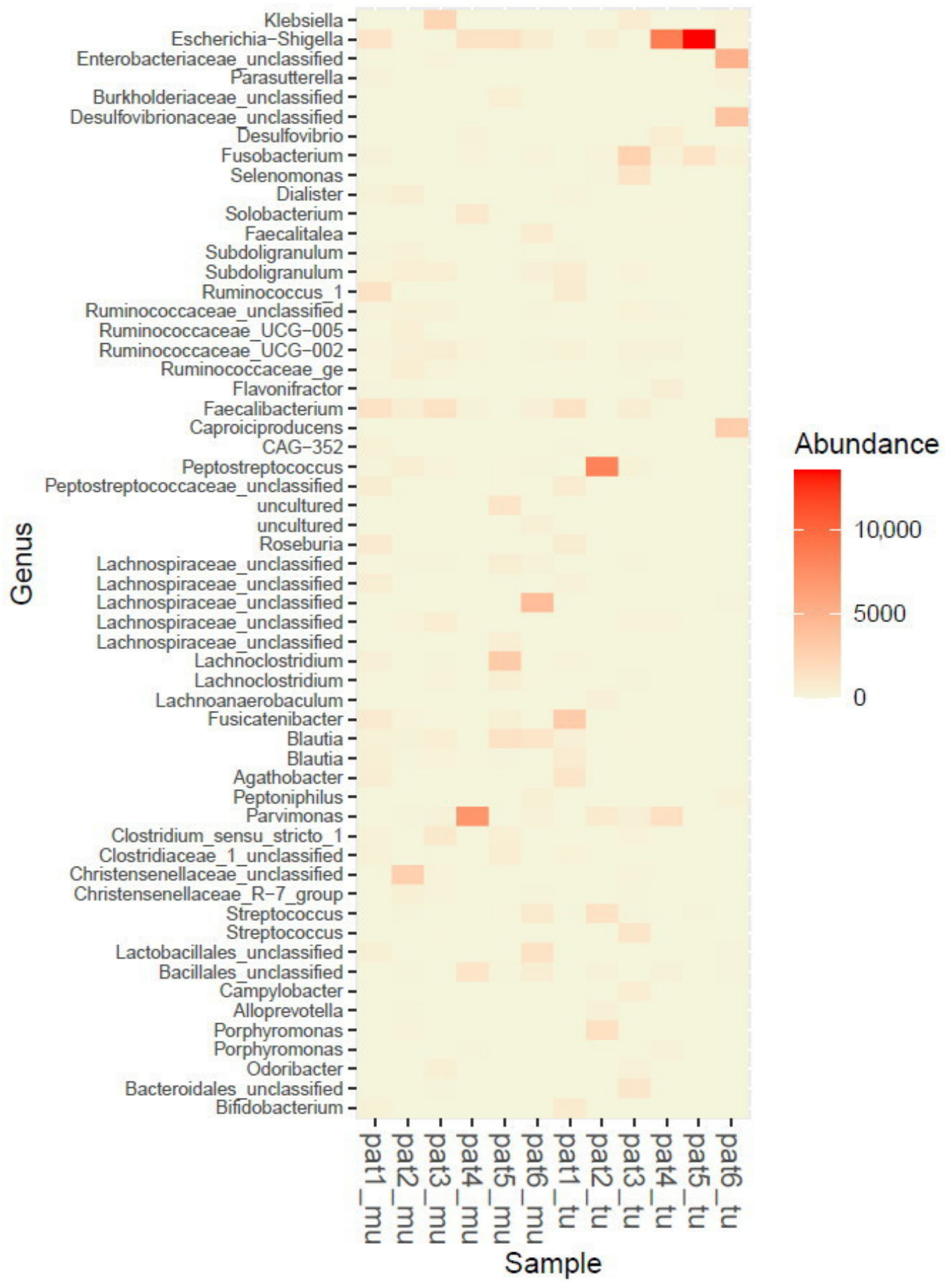

Figure 3. Heatmap of abundance at the genus level for all samples (pat = patient, $m u=$ mucosa, $\mathrm{tu}=$ tumor samples). 
A

A
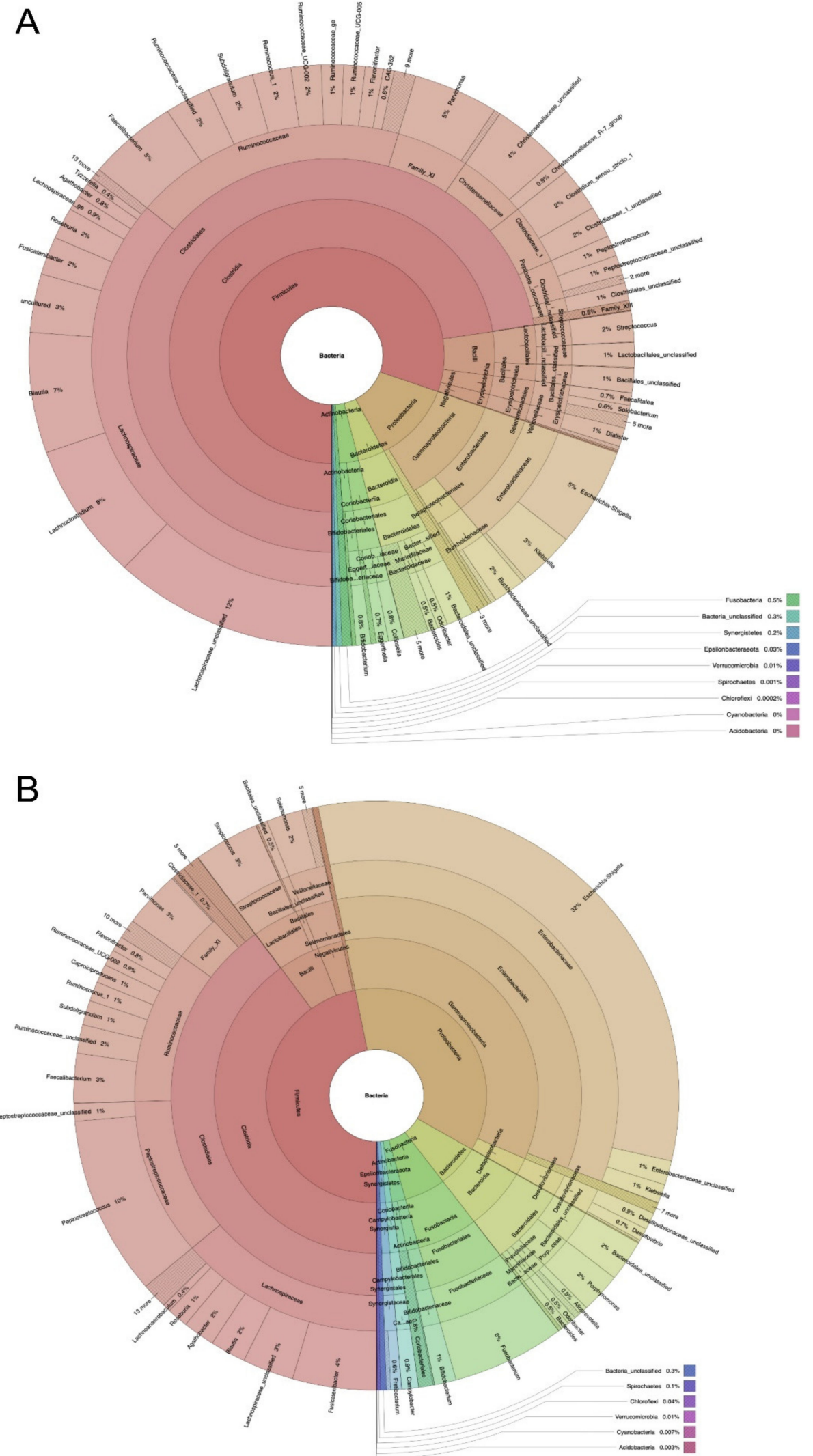

Figure 4. Krona diagrams for illustration of abundance of different OTUs at the genus level (A mucosa, B tumor). 


\section{Discussion}

In CRC, the causative role of microbiota in tumorigenesis and progression is widely accepted $[11,12,20,21]$. However, despite the significant impact of microbiota on CRC development and progression as well as outcomes after colorectal surgery, the role of the microbiome and its interaction with the host is still not fully understood and requires further research. Several studies revealed associations between the colonization of specific species and tumor-specific characteristics such as lymph node metastasis and gene mutations (summarized by Chen et al. [20]). Comparisons of taxonomic groups based on phyla of healthy people and CRC patients revealed differences in the abundance of Proteobacteria, Bacteroidetes, Actinobacteria, Firmicutes and (to the greatest extent) Fusobacteria [20]. Furthermore, some Escherichia coli strains harboring the pks-island (Colibactin gene), enterotoxigenic Bacteroides fragilis and Fusobacterium nucleatum showed the ability to induce a proinflammatory microenvironment, cell proliferation and even direct DNA damage [11,12,22-25].

As gut microbiota significantly contribute to immune function and the restoration of body integrity in healing processes, its impact on wound and anastomotic healing after surgery is under evaluation $[26,27]$. There are complex interactions between gut microbiota and essential cytoprotective mechanisms, bacterial metabolites as well as the immune system. The restoration of a healthy intestinal microbiome following colorectal surgery is depending on multiple perioperative variables such as extent of surgical trauma, perioperative use of antibiotics, bowel preparation and possibility of oral nutrition $[19,26]$. The use of minimally invasive surgical technique, limited use of antibiotics and fast return to oral intake of intake of unprocessed food may have beneficial effects on the intestinal microbiome, whereas refaunation by pathogenic bacteria like Serratia spp., Enterococcus spp. and Pseudomonas spp. following colorectal surgery may significantly increase the risk of anastomotic leakage and surgical site infections as well as sepsis $[10,26]$.

Given the relevance of the microbial profile in CRC and its impact on short-term outcome following colorectal surgery, the mechanisms of interaction between the microbiota and the gut epithelium as well as mucosa-associated immune tissue highlight the importance of microbiome analysis of the mucosal microbial compartment. The results of the present work show that microbiome profiling can be successfully conducted from different compartments and tissue sites, even after long-term storage of snap-frozen samples. In order to choose the right sample type for microbiome studies, one must take into account the differences in the composition of different microbial compartments and tissue sites. We have shown that these sample types could be carefully chosen from samples stored in a comprehensive biobank in a retrospective manner depending on the specific scientific aim.

As fecal samples (luminal compartment) are easy to collect in a non-invasive manner, they are currently widely used for microbiome profiling in association studies, especially for colorectal cancer $[11,12]$. Taking into account differences in microbial patterns between different anatomical locations of the colon not only in CRC, but even healthy individuals, the fecal microbiome appears to be more appropriate for an use as biomarkers as it is representing the overall microbial composition of the colonic microenvironment $[16,28]$. However, the fecal microbiota are more inconsistent due to a higher impact of environmental factors like diet or life-style on the luminal micromilieu [25,29]. The mucosal microbiota are more stable, but can only be analyzed from biopsies or surgical specimen; they are located at the direct interface with the gut epithelium and consequently, are more closely involved in immune processes [25,29-31]. Therefore, the mucosal microbiota play an essential role in chronic inflammation and tumorigenesis in CRC due to their direct interaction with epithelium and immune cells at the gut-mucosa barrier $[11,12,22,25]$. Codling et al. revealed that the intra-individual similarity between luminal and mucosal compartments in a larger cohort was in a range of $84 \%$ [29]. In the present work, we show some variations in phylum level abundance between the compartments without relevant significance in diversity measures or weighted/unweighted UniFrac analysis. 
Comparing the microbiome profiles of tumor to off-tumor-site tissues, we observed a significant reduction in $\alpha$-diversity indices for tumor tissue, which is consistent to recent publications and reflects the intestinal dysbiosis associated with CRC [32]. Furthermore, we could detect some variation in phylum level abundance. Actinobacteria were significantly more abundant in samples of normal mucosa, whereas a non-significant higher abundance of Fusobacteria was observed in tumor tissue samples. These results are in accordance to previously published data $[12,17,22,24,25]$ and supporting the role of Fusobacterium nucleatum as a "passenger" in the tumorigenesis of CRC according to the widely accepted "driver-passenger mode" proposed by Tjalsma et al. [33].

In general, our microbiome profiling results are comparable to other available data using samples from healthy controls and patients with CRC. The predominant phyla were Firmicutes, Proteobacteria and Bacteroidetes, whereas Actinobacteria and Fusobacteria were less abundant [20,24,25]. Recently published data examined the microbial niches along the gastrointestinal tract in German healthy individuals and could demonstrate differences especially between upper and lower gastrointestinal tract and even between the different anatomical regions of the colon and also compared to fecal samples [28]. The predominant phyla in the colon and feces were Firmicutes, Bacteroidetes, Proteobacteria and much less abundant Fusobacteria [28]. An analysis of mucosa-associated microbiome profiles in colorectal cancer samples showed that Firmicutes, Bacteroidetes and Fusobacteria were the most abundant phyla and Proteobacteria were less common in these samples [24]. The $\alpha$-diversity measures like Shannon or Simpson indices show some variations in the available data due to methodical and maybe even regional differences [17], but are consistently showing reduced $\alpha$-diversity levels in CRC in accordance to evident dysbiosis as a main finding in microbial patterns of CRC samples [22,24,25]. Therefore, compared to other available data and taking into account regional and inter-individual differences in abundance of phyla and other taxonomic levels, our technique of sampling, DNA extraction, DNA amplification, sequencing and bioinformatic analysis revealed valid microbiome profiles of the different compartments and tissue samples $[16,24,25,28]$.

In addition, the results of the present work show that valid microbiome profiling even from long-term stored snap frozen samples can be successfully conducted from different compartments and tissue sites. The storage duration of our samples did not significantly affect the number of high-quality DNA reads. The samples were stored at $-80{ }^{\circ} \mathrm{C}$ for a mean of five years, some of them for longer than eight years. Nonetheless, it was possible to perform high-quality microbiome profiling on these samples. All samples were snap frozen in liquid nitrogen with feasibly short ischemia times according to established protocols and then stored at our biobank facilities in concordance with previously published recommendations [34,35]. An established high-volume colorectal tissue repository based on an ethical and legal framework $[36,37]$ therefore represents a suitable base for microbiome research.

The limitations of our work include the number of samples and some variances in biobank and analysis processes. Samples from surgical specimens were immediately collected after removal of the specimen from the surgical field during surgery, but some factors such as warm ischemia or perioperative antibiotic prophylaxis might differ between samples. In this analysis we did not include samples of rectal cancer tissues as in rectal cancer surgery more confounding factors would have had an impact on microbiome analysis due to especially mechanical bowel preparation and neoadjuvant radio-chemotherapy. The use of samples originating mostly of the proximal colon without perioperative use of mechanical bowel preparation allowed us for maintaining an as homogeneous as possible cohort and reduce gross interindividual differences as well as evident differences between anatomical sample sites [16,28].

A comparison to microbiome profiles from stool and tissue samples of other available studies indicates that our results might be of some external validity $[20,24,25,29]$. Despite high inter-individual differences in the gut microbiome, the results of many microbiome studies are limited by small sample sizes and this limitation is also inherent in our study. Nonetheless, our work provides the basis for future work by proving that a retrospective approach assessing a high number of stored samples is feasible and valid microbiome profiles can be generated using long term stored samples. 


\section{Materials and Methods}

\subsection{Ethical Framework}

This study was approved by the Ethics Committee of the Faculty of Medicine, LudwigMaximilians-University (LMU), Munich, Germany. Double-coded tissue and stool samples as well as corresponding procedural and clinical data used in this study were provided by the Biobank of the Department of General, Visceral and Transplant Surgery, LMU, under the administration of the Human Tissue and Cell Research (HTCR $\left.{ }^{\circledR}\right)$ Foundation. The framework of the HTCR Foundation includes obtaining written informed consent from all donors and has been approved by the Ethics Committee of the Faculty of Medicine, LMU, Munich, Germany, (approval number 025-12) as well as the Bavarian State Medical Association (approval number 11142), Germany [36].

\subsection{Study Design and Sample Collection}

From patients undergoing right or left hemicolectomy for colorectal cancer, gut microbiome $16 \mathrm{~S}$ rRNA gene-based microbiome analysis was performed in two independent trial steps. After initial macroscopic examination of the specimen by an expert pathologist, tissue for sample collection was transferred to the biobank's laboratory. Samples from six donors from different sample sites such as feces (luminal compartment), mucosa samples and mucosal scrapings (mucosal compartment) were prospectively collected in the first trial step (step 1) between March and July 2018. Samples were stored snap-frozen using DNA free Eppendorf tubes (2.0 mL DNA LoBind Tubes, PCR clean, Eppendorf, Hamburg, Germany) at $-80{ }^{\circ} \mathrm{C}$. In a second trial step (step 2), microbiome profiling of samples of right or transverse colon from different tissue sites (tumor or normal adjacent mucosa from the same surgical specimen) was performed to assess the mucosal microbiome of tumor and normal adjacent mucosa as well as to investigate the influence of varying storage durations. Therefore, snap-frozen tissue samples of six further donors were used, which were stored at $-80^{\circ} \mathrm{C}$ at the biobank between 2010 and $2018[34,36,37]$.

\subsection{DNA Extraction, Amplification and Sequencing}

Each sample was suspended in $500 \mu \mathrm{L}$ of extraction buffer $(200 \mathrm{mM}$ Tris pH 8.0, $200 \mathrm{mM} \mathrm{NaCl}$, $20 \mathrm{mM}$ EDTA), $210 \mu \mathrm{L}$ of $20 \%$ SDS, $500 \mu \mathrm{L}$ of a mix of phenol:chloroform:isoamyl alcohol (24:24:1) and $500 \mu \mathrm{L}$ of zirconia/silica beads $(0.1 \mathrm{~mm}$ diameter). Lysis of bacterial cells was obtained by mechanical disruption with a bead beater $\left(50 \mathrm{~s}^{-1}\right)$ for $4 \mathrm{~min}$, followed by DNA extraction in phenol:chloroform:isoamyl alcohol and precipitation with ethanol. The isolated DNA was resuspended in $10 \mathrm{mM}$ Tris buffer and purified with NucleoSpin gDNA clean-up columns (Macherey-Nagel, Düren, Germany) in a final elution volume of $50 \mu \mathrm{L}$. The extracted DNA was amplified by PCR using multiplexed 8 forward x 12 reverse-primer, specific for the variable V3-4 regions of the 16S rDNA gene (5'-CCTACGGGNBGCASCAG-3' and 5'-GACTACNVGGGTAT CTAATCC-3'). The amplicons were purified using the Agencourt AMPure XP PCR Purification system (Cat: A63880, Beckman Coulter, Krefeld, Germany). Library quality control and DNA sequencing on the Illumina MiSeq v.3 platform (300-bp paired-end runs) were performed at Eurofins Genomics (Ebersberg, Germany).

\subsection{Bioinformatics and Statistical Analysis}

The sequencing output was processed and joined reads were then quality filtered during demultiplexing using QIIME v1.8 to retain only high-quality reads [38]. Only reads that possessed the full barcode free of errors, were longer than $200 \mathrm{bp}$ after removal of the variability region as well as quality trimming and had a minimum of $30 \mathrm{bp}$ overlap without mismatches during merging of paired-end reads were kept for further analysis. 
Chimeric sequences were removed with USEARCH v6.1 (step 1) [39] and vsearch (step 2) [38]. respectively. Open-reference OTU clustering and taxonomic assignment using paired-end sequencing reads was done against the Silva database release 132 at the $97 \%$ similarity level using mothur v1.43.0 [40]. Further analysis was performed using $R$ studio (version 1.8 and phyloseq package [41]) as well as SPSS (IBM, Armonk, NY, USA; Version 25). For analysis of diversity measures, ordination and non-parametric analyses, data were sub-sampled to the sample with the lowest number of reads. For diversity analysis, $\alpha$-diversity measures including Chao1 richness, abundance-based coverage estimators (ACE) and diversity indices Shannon and Simpson Index were calculated. For estimation of differences between groups, weighted and unweighted UniFrac were calculated as distance metrics [42,43]. Based on UniFrac distances, principal coordinate analysis (PCoA) was performed for graphic illustration. Permutational multivariate analysis of variance (PERMANOVA) was preformed using $R$ vegan [44] package (ADONIS function) for analysis of dissimilarities between groups based on UniFrac distance metrics [42,43]. Heat maps and bar as well as box plots were generated using the $R$ ggplot2 package [45]. For comparison of diversity measures and abundance of most abundant phyla between groups, Wilcoxon rank sum test and Friedman two-way analysis of variance were used. Pearson correlation was used to analyze the relationship between genomic DNA concentration after extraction, amplicon concentration, storage conditions and number of high-quality reads per sample. Krona diagrams of composition of local microbial environment were generated using Krona tools [46]. $p$ values $<0.05$ were considered statistically significant.

\section{Conclusions}

The results of the present work show that microbiome analysis, even from long-term stored snap frozen samples of a colorectal cancer biorepository, can be successfully conducted from different compartments and tissue sites. For several scientific study aims, this may be a feasible approach avoiding the time-consuming prospective collection of samples to perform association studies based not only on microbiome research but also to assess metabolomics profiles in CRC [23].

Author Contributions: Conceptualization, U.W., D.G., B.S. and T.S.S.; methodology, U.W., D.G., L.M.J., S.S., F.K., S.M.L.L., A.V.B., B.S. and T.S.S.; software, U.W., D.G., L.M.J., S.S., B.S.; validation, U.W., F.K., M.I., S.M.L.L., H.N., A.V.B., J.A., B.S. and T.S.S.; formal analysis, U.W., D.G., L.M.J., S.S. and T.S.S.; investigation, U.W., D.G., L.M.J., S.S., B.S. and T.S.S.; resources, U.W., D.G., H.N., A.V.B., J.A., J.W., B.S. and T.S.S.; data curation, U.W., D.G., L.M.J., S.S. and T.S.S.; writing — original draft preparation, U.W., D.G., L.M.J., S.S. and T.S.S.; writing-review and editing, F.K., M.I., S.M.L.L., H.N., A.V.B., J.A., J.W. and B.S.; visualization, U.W., D.G., B.S. and T.S.S; supervision, A.V.B., J.A., J.W., B.S. and T.S.S.; project administration, U.W., D.G., B.S. and T.S.S.; funding acquisition, B.S. and T.S.S. All authors have read and agreed to the published version of the manuscript.

Funding: This research was funded by the Center for Gastrointestinal Microbiome Research (CEGIMIR), German Center of Infection Research (DZIF).

Acknowledgments: This study was supported by the Human Tissue and Cell Research $\left(\mathrm{HTCR}^{\circledR}\right)$ Foundation, a non-profit foundation regulated by German civil law, which facilitates research with human tissue through the provision of an ethical and legal framework for sample collection and the Center for Gastrointestinal Microbiome Research (CEGIMIR).

Conflicts of Interest: The authors declare no conflict of interest.

\section{References}

1. Allen, J.; Sears, C.L. Impact of the gut microbiome on the genome and epigenome of colon epithelial cells: Contributions to colorectal cancer development. Genome Med. 2019, 11, 1-18. [CrossRef]

2. Lynch, S.V.; Pedersen, O. The Human Intestinal Microbiome in Health and Disease. N. Engl. J. Med. 2016, 375, 2369-2379. [CrossRef] [PubMed]

3. Qin, J.; Li, Y.; Cai, Z.; Li, S.; Zhu, J.; Zhang, F.; Liang, S.; Zhang, W.; Guan, Y.; Shen, D.; et al. A metagenomewide association study of gut microbiota in type 2 diabetes. Nat. Cell Biol. 2012, 490, 55-60. [CrossRef] [PubMed] 
4. Schirmer, M.; Franzosa, E.A.; Lloyd-Price, J.; McIver, L.J.; Schwager, R.; Poon, T.W.; Ananthakrishnan, A.N.; Andrews, E.; Barron, G.; Lake, K.; et al. Dynamics of metatranscription in the inflammatory bowel disease gut microbiome. Nat. Microbiol. 2018, 3, 337-346. [CrossRef] [PubMed]

5. Feng, Q.; Liang, S.; Jia, H.; Stadlmayr, A.; Tang, L.; Lan, Z.; Zhang, D.; Xia, H.; Xu, X.; Jie, Z.; et al. Gut microbiome development along the colorectal adenoma-carcinoma sequence. Nat. Commun. 2015, 6, 6528. [CrossRef] [PubMed]

6. Whiteside, T.L.; Shoenfeld, Y. The microbiome in autoimmune diseases. Clin. Exp. Immunol. 2019, 195, 74-85. [CrossRef]

7. Barrington, W.T.; Lusis, A.J. Atherosclerosis: Association between the gut microbiome and atherosclerosis. Nat. Rev. Cardiol. 2017, 14, 699-700. [CrossRef]

8. Lazar, V.; Ditu, L.-M.; Pircalabioru, G.G.; Gheorghe, I.; Curutiu, C.; Holban, A.M.; Picu, A.; Petcu, L.; Chifiriuc, M.C. Aspects of Gut Microbiota and Immune System Interactions in Infectious Diseases, Immunopathology, and Cancer. Front. Immunol. 2018, 9, 1830. [CrossRef]

9. Belizário, J.E.; Napolitano, M. Human microbiomes and their roles in dysbiosis, common diseases, and novel therapeutic approaches. Front. Microbiol. 2015, 6, 1050. [CrossRef]

10. Gaines, S.; Shao, C.; Hyman, N.; Alverdy, J.C. Gut microbiome influences on anastomotic leak and recurrence rates following colorectal cancer surgery. BJS 2018, 105, e131-e141. [CrossRef]

11. Mizutani, S.; Yamada, T.; Yachida, S. Significance of the gut microbiome in multistep colorectal carcinogenesis. Cancer Sci. 2020, 111, 766-773. [CrossRef] [PubMed]

12. Wirbel, J.; Pyl, P.T.; Kartal, E.; Zych, K.; Kashani, A.; Milanese, A.; Fleck, J.S.; Voigt, A.Y.; Palleja, A.; Ponnudurai, R.; et al. Meta-analysis of fecal metagenomes reveals global microbial signatures that are specific for colorectal cancer. Nat. Med. 2019, 25, 679-689. [CrossRef] [PubMed]

13. Gethings-Behncke, C.; Coleman, H.G.; Jordao, H.W.; Longley, D.B.; Crawford, N.; Murray, L.J.; Kunzmann, A.T. Fusobacterium nucleatum in the Colorectum and Its Association with Cancer Risk and Survival: A Systematic Review and Meta-analysis. Cancer Epidemiol. Biomark. Prev. 2020, 29, 539-548. [CrossRef] [PubMed]

14. Arthur, J.C.; Perez-Chanona, E.; Muhlbauer, M.; Tomkovich, S.; Uronis, J.M.; Fan, T.J.; Campbell, B.J.; Abujamel, T.; Dogan, B.; Rogers, A.B.; et al. Intestinal inflammation targets cancer-inducing activity of the microbiota. Science 2012, 338, 120-123. [CrossRef] [PubMed]

15. Klimesova, K.; Zakostelska, Z.J.; Tlaskalova-Hogenova, H. Oral Bacterial and Fungal Microbiome Impacts Colorectal Carcinogenesis. Front. Microbiol. 2018, 9, 774. [CrossRef]

16. Flemer, B.; Lynch, D.B.; Brown, J.M.R.; Jeffery, I.B.; Ryan, F.J.; Claesson, M.J.; O’Riordain, M.; Shanahan, F.; O'Toole, P.W. Tumour-associated and non-tumour-associated microbiota in colorectal cancer. Gut 2017, 66, 633-643. [CrossRef]

17. He, Y.; Wu, W.; Zheng, H.-M.; Li, P.; McDonald, D.; Sheng, H.-F.; Chen, M.-X.; Chen, Z.-H.; Ji, G.-Y.; Zheng, Z.-D.-X.; et al. Regional variation limits applications of healthy gut microbiome reference ranges and disease models. Nat. Med. 2018, 24, 1532-1535. [CrossRef]

18. Zeller, G.; Tap, J.; Voigt, A.Y.; Sunagawa, S.; Kultima, J.R.; Costea, P.I.; Amiot, A.; Böhm, J.; Brunetti, F.; Habermann, N.; et al. Potential of fecal microbiota for early-stage detection of colorectal cancer. Mol. Syst. Biol. 2014, 10, 766. [CrossRef]

19. Koliarakis, I.; Athanasakis, E.; Sgantzos, M.; Mariolis-Sapsakos, T.; Xynos, E.; Chrysos, E.; Souglakos, J.; Tsiaoussis, J. Intestinal Microbiota in Colorectal Cancer Surgery. Cancers 2020, 12, 3011. [CrossRef]

20. Chen, Y.; Yang, Y.; Gu, J. Clinical Implications of the Associations Between Intestinal Microbiome and Colorectal Cancer Progression. Cancer Manag. Res. 2020, 12, 4117-4128. [CrossRef]

21. Drewes, J.L.; Housseau, F.; Sears, C.L. Sporadic colorectal cancer: Microbial contributors to disease prevention, development and therapy. Br. J. Cancer 2016, 115, 273-280. [CrossRef] [PubMed]

22. Brennan, C.A.; Garrett, W.S. Gut Microbiota, Inflammation, and Colorectal Cancer. Annu. Rev. Microbiol. 2016, 70, 395-411. [CrossRef] [PubMed]

23. Kim, M.; Vogtmann, E.; Ahlquist, D.A.; Devens, M.E.; Kisiel, J.B.; Taylor, W.R.; White, B.A.; Hale, V.L.; Sung, J.; Chia, N.; et al. Fecal Metabolomic Signatures in Colorectal Adenoma Patients Are Associated with Gut Microbiota and Early Events of Colorectal Cancer Pathogenesis. mBio 2020, 11. [CrossRef] [PubMed]

24. Xu, K.; Jiang, B. Analysis of Mucosa-Associated Microbiota in Colorectal Cancer. Med. Sci. Monit. 2017, 23, 4422-4430. [CrossRef] [PubMed] 
25. Yoon, H.; Kim, N.; Park, J.H.; Kim, Y.S.; Lee, J.; Kim, H.W.; Choi, Y.J.; Shin, C.M.; Park, Y.S.; Lee, N.H.; et al. Comparisons of Gut Microbiota Among Healthy Control, Patients with Conventional Adenoma, Sessile Serrated Adenoma, and Colorectal Cancer. J. Cancer Prev. 2017, 22, 108-114. [CrossRef]

26. Alverdy, J.C.; Hyoju, S.K.; Weigerinck, M.; Gilbert, J.A. The gut microbiome and the mechanism of surgical infection. BJS 2017, 104, e14-e23. [CrossRef]

27. Guyton, K.; Alverdy, J.C. The gut microbiota and gastrointestinal surgery. Nat. Rev. Gastroenterol. Hepatol. 2016, 14, 43-54. [CrossRef] [PubMed]

28. Vasapolli, R.; Schütte, K.; Schulz, C.; Vital, M.; Schomburg, D.; Pieper, D.H.; Vilchez-Vargas, R.; Malfertheiner, P. Analysis of Transcriptionally Active Bacteria throughout the Gastrointestinal Tract of Healthy Individuals. Gastroenterology 2019, 157, 1081-1092.e3. [CrossRef]

29. Codling, C.; O'Mahony, L.; Shanahan, F.; Quigley, E.M.M.; Marchesi, J.R. A Molecular Analysis of Fecal and Mucosal Bacterial Communities in Irritable Bowel Syndrome. Dig. Dis. Sci. 2009, 55, 392-397. [CrossRef]

30. Parthasarathy, G.; Chen, J.; Chen, X.; Chia, N.; O'Connor, H.M.; Wolf, P.G.; Gaskins, H.R.; Bharucha, A.E. Relationship between Microbiota of the Colonic Mucosa vs. Feces and Symptoms, Colonic Transit, and Methane Production in Female Patients with Chronic Constipation. Gastroenterology 2016, 150, 367-379.e1. [CrossRef]

31. Arumugam, M.; Raes, J.; Pelletier, E.; Le Paslier, D.; Yamada, T.; Mende, D.R.; Fernandes, G.R.; Tap, J.; Bruls, T.; Batto, J.M.; et al. Enterotypes of the human gut microbiome. Nature 2011, 473, 174-180. [CrossRef] [PubMed]

32. Sánchez-Alcoholado, L.; Ramos-Molina, B.; Otero, A.; Laborda-Illanes, A.; Ordóñez, R.; Medina, J.A.; Gomez-Millan, J.; Queipo-Ortuño, M.I. The Role of the Gut Microbiome in Colorectal Cancer Development and Therapy Response. Cancers 2020, 12, 1406. [CrossRef] [PubMed]

33. Tjalsma, H.; Boleij, A.; Marchesi, J.R.; Dutilh, B.E. A bacterial driver-passenger model for colorectal cancer: Beyond the usual suspects. Nat. Rev. Genet. 2012, 10, 575-582. [CrossRef] [PubMed]

34. Lee, S.M.L.; Schelcher, C.; Gashi, S.; Schreiber, S.; Thasler, R.M.K.; Jauch, K.-W.; Thasler, W.E. RNA Stability in Human Liver: Comparison of Different Processing Times, Temperatures and Methods. Mol. Biotechnol. 2013, 53, 1-8. [CrossRef]

35. Lee, S.M.L.; Schelcher, C.; Thasler, R.; Schiergens, T.S.; Thasler, W.E. Pre-Analytical Determination of the Effect of Extended Warm or Cold Ischaemia on RNA Stability in the Human Ileum Mucosa. PLoS ONE 2015, 10, e0138214. [CrossRef] [PubMed]

36. Thasler, W.E.; Weiss, T.S.; Schillhorn, K.; Stoll, P.-T.; Irrgang, B.; Jauch, K.-W. Charitable State-Controlled Foundation Human Tissue and Cell Research: Ethic and Legal Aspects in the Supply of Surgically Removed Human Tissue for Research in the Academic and Commercial Sector in Germany. Cell Tissue Bank. 2003, 4, 49-56. [CrossRef]

37. Thasler, W.E.; Thasler, R.M.K.; Schelcher, C.; Jauch, K.-W. Biobanking for research in surgery: Are surgeons in charge for advancing translational research or mere assistants in biomaterial and data preservation? Langenbeck's Arch. Surg. 2013, 398, 487-499. [CrossRef]

38. Rognes, T.; Flouri, T.; Nichols, B.; Quince, C.; Mahé, F. VSEARCH: A versatile open source tool for metagenomics. PeerJ 2016, 4, e2584. [CrossRef]

39. Edgar, R.C. Search and clustering orders of magnitude faster than BLAST. Bioinformatics 2010, 26, $2460-2461$. [CrossRef]

40. Quast, C.; Pruesse, E.; Yilmaz, P.; Gerken, J.; Schweer, T.; Yarza, P.; Peplies, J.; Glöckner, F.O. The SILVA ribosomal RNA gene database project: Improved data processing and web-based tools. Nucleic Acids Res. 2013, 41, D590-D596. [CrossRef]

41. McMurdie, P.J.; Holmes, S. phyloseq: An R package for reproducible interactive analysis and graphics of microbiome census data. PLoS ONE 2013, 8, e61217. [CrossRef] [PubMed]

42. Lozupone, C.; Knight, R. UniFrac: A New Phylogenetic Method for Comparing Microbial Communities. Appl. Environ. Microbiol. 2005, 71, 8228-8235. [CrossRef] [PubMed]

43. Chang, Q.; Luan, Y.; Sun, F. Variance adjusted weighted UniFrac: A powerful beta diversity measure for comparing communities based on phylogeny. BMC Bioinform. 2011, 12, 118. [CrossRef] [PubMed]

44. Oksanen, J.; Blanchet, F.G.; Kindt, R.; Legendre, P.; Minchin, P.R.; O’hara, R.B.; Simpson, G.L.; Solymos, P.; Stevens, M.H.H.; Wagner, H. vegan: Community Ecology Package R Package Version 2.5-6. 2019. Available online: https://CRAN.R-project.org/package=vegan (accessed on 12 October 2020). 
45. Wickham, H. Ggplot2. Elegant Graphics for Data Analysis; Springer: Berlin/Heidelberg, Germany, 2016.

46. Ondov, B.D.; Bergman, N.H.; Phillippy, A.M. Interactive metagenomic visualization in a Web browser. BMC Bioinform. 2011, 12, 385. [CrossRef]

Publisher's Note: MDPI stays neutral with regard to jurisdictional claims in published maps and institutional affiliations.

(C) 2020 by the authors. Licensee MDPI, Basel, Switzerland. This article is an open access article distributed under the terms and conditions of the Creative Commons Attribution (CC BY) license (http://creativecommons.org/licenses/by/4.0/). 